\title{
Monitoring and Reporting of Adverse Drug Reactions Due to Cardiovascular Drugs in Patients Admitted to a Secondary Care Hospital in Northern Emirate- A Prospective Surveillance Study
}

\author{
Tarun Wadhwa', Samar Gamal El Sheikh², Padma G.M. Rao ${ }^{3}$ \\ 1'Department of Clinical Pharmacy and Pharmacology, RAK College of Pharmaceutical Sciences, RAK Medical and Health Sciences \\ University, Ras Al Khaimah, UAE. \\ ${ }^{2}$ Clinical Pharmacy Department at Al Ahrar Teaching Hospital, Zagazig, EGYPT. \\ ${ }^{3}$ RAK College of Pharmaceutical Sciences, RAK Medical and Health Sciences University, Ras Al Khaimah, UAE.
}

\begin{abstract}
Context: Adverse drug reactions (ADRs) can result in a number of consequences, ranging from allergic reactions to permanent harm thereby causing morbidity and mortality leading to an increase in the health care costs. Aims: The main objective of the present study was to assess the prevalence of adverse drug reactions due to cardiovascular drugs in patients admitted to a secondary care hospital. Material and Methods: This was a prospective surveillance study which included adult patients of either gender who were hospitalized and prescribed with at least one cardiovascular drug. Patients were monitored from the day of admission till the day of discharge for the occurrence of ADRs due to cardiovascular drugs by attending clinical meetings, ward rounds, reviewing patients' medical records and electronic medical records. Results: A total of 309 patients were enrolled in the present study. Among them, 72 patients experienced 109 ADRs accounting for an incidence of $23.3 \%$. Twelve (3.88\%) patients were hospitalized due to ADRs ( $\mathrm{n}=16)$ whereas $60(19.41 \%)$ patients developed ADRs ( $\mathrm{n}=93$ ) during their hospital stay. Male preponderance was observed over female. Patients with age $\geq 60$ years experienced 73 ADRs. The most common drug class implicated in ADRs was observed to be beta blockers $(18 ; 16.5 \%)$ especially bisoprolol (16; 14.68\%). Majority of patients experienced bradycardia (11; 10.09\%) followed by hypotension (9; 8.26\%). Cardiovascular system $(30 ; 27.53 \%)$ was observed to be the most common system. Majority of ADRs were possible $(80 ; 73.39 \%)$ in nature, mild $(53 \%)$ in severity and not preventable $(84.5 \%)$. Conclusion: Intensive approach towards monitoring and reporting of ADRs could help healthcare professionals in minimizing preventable ADRs.
\end{abstract}

Key words: Adverse drug reaction, Cardiovascular drugs, Causality, Pharmacovigilance, Preventability, Severity.

Key messages: Study demonstrated high incidence of adverse drug reactions in patients on cardiovascular drugs. This triggers an alarming signal for vigilant monitoring of patients to prevent further recurrence

\section{INTRODUCTION}

Adverse drug reactions (ADRs) are one of the major problems in health care system. Till date, there is no consensus on the definition of an ADR but the World Health Organization (WHO) proposed it as "any response to a drug which is noxious and un intended and which occurs at doses normally used in human for therapy, prophylaxis, or a diagnosis of diseases or for modification of physiological function". Recent epidemiological studies estimated that adverse drug reactions (ADRs) are fourth to sixth leading cause of death. ${ }^{1}$
Adverse drug reactions are observed in day to day clinical practice, but estimation of the true incidence of ADRs is difficult and highly variable among countries all over the world. Prevalence of ADRs varies from $14.7 \%$ in UK to $16.2 \%$ in India. ${ }^{2-3}$ The incidence of ADRs leading to hospital admissions ranges from 2.4 to $12 \%$ while incidence of ADRs during hospital stay ranges from 0.05 to 0.19 $\%{ }^{4-5}$ Fatality due to ADRs was reported to be around $0.3 \%{ }^{6}$

Adverse drug reactions can result in a number of consequences, ranging from allergic reactions to permanent harm thereby
DOI: 10.5530/ijopp.11.2.16

\section{Address for} correspondence: Dr. Tarun Wadhwa, Assistant Professor, Department of Clinical Pharmacy \& Pharmacology, RAK College of Pharmaceutical Sciences, RAK Medical \& Health Sciences University, Ras AI Khaimah, UAE.

Phone no: +971 503747342

Email Id: tarun@rakmhsu.ac.ae

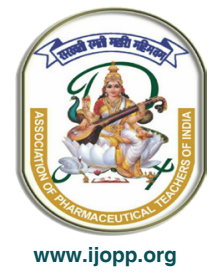


causing morbidity and mortality leading to an increase in the health care costs. ${ }^{7}$ It has been reported that over 770,000 people are injured or die each year in hospitals from ADRs, which may account for $\$ 5.6$ million of healthcare costs. ${ }^{8}$

As projected, patients who experience ADRs have longer and more expensive hospitalization. It has been reported that patients who experienced ADRs were hospitalized on an average of 1 to 5 days longer than patients who did not suffer ADRs, with additional costs of up to $\$ 9,000$. $^{1}$ In another study, it was reported that ADRs increase the length of hospital stay by as much as 4.6 days with incremental cost up to $\$ 4,685$.

The increased complexity of drug therapy requires strict vigilance by health care professionals. Hence, it is of utmost importance for healthcare professionals to safeguard their patients from preventable ADRs. This could be achieved by careful monitoring and prompt reporting under the strong and robust system so called pharmacovigilance. Pharmacovigilance is the science and activities relating to detection, assessment, understanding and prevention of adverse effects or any other drug related problems. Pharmacovigilance should not be limited to the reporting of known adverse effects. The scope of pharmacovigilance is broader in its function than that of post-marketing surveillance as it implicates clinical as well as preclinical development of the drug. ${ }^{10}$

According to WHO guidelines (2000), functions of pharmacovigilance are the detection and study of ADRs, measurement of risk and effectiveness of drug use, dissemination of this information and education. ${ }^{11}$

Monitoring of adverse drug reactions could be achieved by applying the following steps: Identifying adverse drug reaction, assessing causality between drug and suspected reaction, documentation of ADR in patient's medical records and the last step is reporting suspected ADRs to pharmacovigilance centers nationally as well as internationally. ${ }^{12}$

According to a recent survey, only six out of thirteen countries in the Middle East possess pharmacovigilance centers. United Arab Emirates (UAE) is one among them but the spontaneous reporting system is still in its infancy stage. Therefore, a systematic way of reporting ADRs is very essential to assess the incidence of ADRs and create awareness among the public. ${ }^{13}$

Many new drugs are being introduced every year and so every health care professional must have knowledge about importance of ADR monitoring and pharmacovigilance activities and care for those patients who are more prone to develop ADRs especially elderly, hypertensive and cardiovascular diseases patients.

Cardiovascular diseases (CVDs) are the leading cause of death according to American Heart Association $(\mathrm{AHA})^{14}$ and account for over a third of all deaths in Australia each year ${ }^{15}$. Between 1990 and 2020, the proportion of worldwide deaths from cardiovascular disease is expected to increase from $28.9 \%$ to $36.3 \% .{ }^{16}$ The ageing population, increasing levels of obesity, lack of physical activity and the increasing number of diabetic patients, will all contribute to the significant impact of cardiovascular disease in the community.

Drugs used for management of cardiovascular diseases are not devoid of adverse effects but could lead to adverse consequences if not monitored properly. With increased number of medicines for cardiac patients, there is a tendency to cause drug related problems such as ADRs, drug-drug Interactions (DDIs) etc.

Cardiovascular drugs have been reported to account for $9 \%$ of medication related visits to clinics. ${ }^{17}$ One in every five cardiac patients is known to experience ADR and $17.9 \%$ of those are preventable, which further insist for intensive monitoring and reporting. ${ }^{18}$

Since cardiovascular diseases are one of the major concerns and cardiac drugs can cause a multitude of ADRs, development of a robust network for detection and reporting of ADRs is of utmost importance. Due to paucity of literature in the Middle East, the present study was planned with a view to monitor and report ADRs in patients, who are on cardiac drugs, admitted to a secondary care hospital located in Ras Al Khaimah, UAE.

The main objective of the present study was to assess the prevalence of adverse drug reactions due to cardiovascular drugs in patients admitted to a secondary care hospital. Other secondary objectives were to identify and categorize ADRs based on clinical parameters and to analyze reported ADRs for causality, severity and preventability based on standard assessment scales.

\section{MATERIAL AND METHODS}

The present study was approved by RAK Medical and Health Sciences University, Research and Ethics Committee and Ras Al Khaimah Research and Ethics Committee. The study was carried out at Ibrahim Bin Hamad Obaidallah Hospital, secondary care hospital located in Ras Al Khaimah, United Arab Emirates, for a period of nine months from September 2013 to May 2014. 
This was a prospective surveillance study which included adult patients of either gender who were hospitalized and prescribed with at least one cardiovascular drug. Patients on drugs other than cardiovascular medications, mentally retarded patients, and pregnant women were excluded from the study.

The present study was initiated with enrollment of patients based on inclusion and exclusion criteria. Patients were monitored from the day of admission till the day of discharge for the occurrence of ADRs due to cardiovascular drugs by attending clinical meetings, ward rounds, reviewing patients' medical records and electronic medical records. If ADR was detected, the case details were documented in the patient profile form and ADR related details in ADR monitoring and documentation form designed for the study purpose. Adverse drug reactions were evaluated for various clinical parameters such as demographics, individual drug implicated, drug class implicated, organ system affected, type of ADRs, risk factors etc. Reported ADRs were also analyzed for causality ${ }^{19}$ severity ${ }^{20}$ and preventability ${ }^{21}$ using standard assessment scales. Data generated from the study was analyzed statistically using SPSS version-18.0. Statistical tests such as student ' $\mathrm{t}$ ' test was used to assess the difference between parameters such as gender; male and female, length of hospital stay between patients with ADRs and without ADRs. ANOVA and Chi square tests were used to identify the association between different age groups. Pearson correlation was used to ascertain association between number of drugs and number of ADRs.

\section{RESULTS}

A total of 309 patients were enrolled in the present study. Among them, 72 patients experienced 109 ADRs accounting for an incidence of $23.3 \%$. Twelve (3.88\%) patients were hospitalized due to ADRs $(n=16)$ whereas $60(19.41 \%)$ patients developed ADRs ( $\mathrm{n}=93)$ during their hospital stay. It was observed that the average length of hospital stays for patients who developed ADRs (10.11 days) was higher than patients without ADRs (6.29 days).

Reported ADRs were evaluated for the following parameters: demographics, drug class implicated, individual drug implicated, suspected ADRs, organ system affected, management and outcome, analysis of ADRs for causality, severity and preventability using standard assessment scales.

\section{Demographics}

Indian Journal of Pharmacy Practice, Vol 11, Issue 2, Apr-Jun, 2018
Out of the 309 patients enrolled in the study, 44 male patients developed 62 ADRs. Among 132 female patients, $28(21.2 \%)$ experienced 47 ADRs. Mean number of ADRs in male and female was $1.4 \pm 0.5$ and $1.6 \pm 0.9$ respectively. There was no significant $(\mathrm{p}=0.183)$ difference in the number of ADRs between male and female (Table 1).

Considering the age group, 45 out of 184 (59.54\%) patients above 60 years of age experienced a total of 73 ADRs with incidence rate of $24.5 \%$ whereas 27 out of $125(40.45 \%)$ patients below 60 years of age experienced 36 ADRs with incidence rate of $21.6 \%$. The difference between the age groups was observed to be statistically insignificant $(\mathrm{p}=0.834)$ (Table 2$)$.

\section{Drug class implicated in ADRs}

The most common drug class implicated in ADRs was observed to be beta blockers (18; 16.5\%). This was followed by Renin-angiotensin-aldosterone system blockers (RAAS blockers) [17; 15.6\%], anti-coagulants (14; $12.84 \%)$, calcium channel blockers $(12 ; 11.01 \%)$, anti-hyperlipidemics $(12 ; 11.01 \%)$, diuretics $(10 ; 9.17 \%)$, anti-anginal agents $(9 ; 8.26 \%)$ and anti-platelet agents (9; $8.26 \%$ ) (Figure 1).

\section{Individual drug implicated in ADRs}

Among the individual drugs, bisoprolol (16; 14.68\%) was the most common drug implicated in causing ADRs. This was followed by amlodipine (10; 9.17\%), warfarin $(9 ; 8.26 \%)$, furosemide $(8 ; 7.34 \%)$, nitroglycerine $(8 ; 7.34 \%)$, atorvastatin $(7 ; 6.42 \%)$ and aspirin $(7 ; 6.42 \%)$. Other drugs involved in causing ADRs were amiodarone, carvedilol and clopidogrel (Table 3).

\begin{tabular}{ccccc}
\multicolumn{2}{c}{ Table 1: Demographics (Gender). } & & \\
Gender & $\begin{array}{c}\text { Total No. of } \\
\text { patients }\end{array}$ & $\begin{array}{c}\text { No. of } \\
\text { patients } \\
\text { with ADRs }\end{array}$ & $\begin{array}{c}\text { No. of } \\
\text { ADRs }\end{array}$ & $\begin{array}{c}\text { Incidence } \\
\text { (\%) }\end{array}$ \\
\hline Male & 177 & 44 & 62 & $24.9^{*}$ \\
Female & 132 & 28 & 47 & 21.2 \\
\hline
\end{tabular}

* 't' student test, $(p=0.183)$.

\section{Table 2: Demographics (Age).}

\begin{tabular}{ccccc}
$\begin{array}{c}\text { Age } \\
\text { group } \\
\text { (Years) }\end{array}$ & $\begin{array}{c}\text { Number of } \\
\text { patients } \\
\text { (A) }\end{array}$ & $\begin{array}{c}\text { Number of } \\
\text { patients with } \\
\text { ADRs (B) }\end{array}$ & $\begin{array}{c}\text { No. of } \\
\text { ADRs }\end{array}$ & $\begin{array}{c}\text { Incidence } \\
(\%) \\
\text { [(B/A) })\end{array}$ \\
\hline$<40$ & 21 & 1 & 1 & 4.8 \\
$40-49$ & 38 & 9 & 13 & 23.7 \\
$50-59$ & 66 & 17 & 22 & 25.8 \\
$\geq 60$ & 184 & 45 & $73^{*}$ & 24.5 \\
\hline
\end{tabular}

*ANOVA test, $(p=0.834)$. 
Drugs with 2 ADRs were tenecteplase, carvedilol, amiodarone, telmisartan and clopidogrel. Drugs with one ADR were adenosine, Lisinopril, Dabigatran, dobutamine, dopamine, and doxazocin.

\section{Suspected adverse drug reactions}

In the present study, majority of patients experienced bradycardia $(11 ; 10.09 \%)$, followed by hypotension (9; $8.26 \%)$, elevated serum creatinine $(9 ; 8.26 \%)$, constipation $(8 ; 7.34 \%)$, electrolyte imbalance $(8 ; 7.34 \%)$, abdominal discomfort $(5 ; 4.59 \%)$, vomiting $(5 ; 4.59 \%)$ and raised International normalized ratio (INR) [5; 4.59\%] (Table 4).

Reactions with 2 ADRs were dizziness, diarrhea, nausea, epigastric pain, oral candidiasis, ecchymosis, haematuria and haemoptysis.

\section{Organ system affected by ADRs}

On evaluation of organ system affected, cardiovascular system $(30 ; 27.53 \%)$ was observed to be the most common system. This was followed by gastrointestinal $(27 ; 24.77 \%)$, renal $(11 ; 10.09 \%)$, electrolyte $(8 ; 7.34 \%)$, central nervous system $(7 ; 6.42 \%)$, respiratory $(6 ; 5.5 \%)$ and haematological system $(6 ; 5.5 \%)$. Other systems such as hepatic $(4 ; 3.67 \%)$, dermatological $(3 ; 2.75 \%)$, and musculoskeletal $(2 ; 1.83 \%)$ were also affected (Figure 2).

\section{Management and outcome of ADRs}

The drug was withdrawn in $45(41.28 \%)$ suspected ADRs and 37 (33.95\%) ADRs were managed by careful monitoring of patients without changing the suspected drug. Dose was altered in $11(10.09 \%)$ ADRs, while suspected drug was withheld in $10(9.17 \%)$ ADRs.

In majority of ADRs, complete recovery $(60 ; 83.33 \%)$ was observed whereas, reaction was continued in $6(5.5 \%)$ of cases (Table 5).

\section{Analysis of adverse drug reactions}

Adverse drug reactions were analyzed for causality, severity and preventability based on standard assessment scales like WHO probability scale, Hartwig et al. scale and modified Schumock and Thornton scale.

\section{Causality assessment}

On causality assessment, it was observed that majority $(80 ; 73.39 \%)$ of reactions were possible in nature, followed by probable $(19 ; 17.43 \%)$, certain $(6 ; 5.50 \%)$ and unlikely (4; 3.67\%) (Table 6$)$.

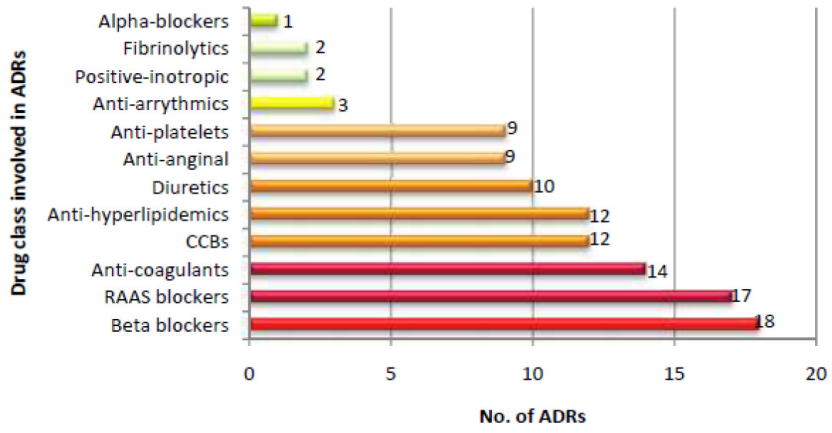

Figure 1: Drug class implicated in ADRs.

Table 3: Individual drug implicated in ADRs

\begin{tabular}{cc} 
Individual drug & No. (\%) of ADRs \\
\hline Bisoprolol & $16(14.68)$ \\
Amlodipine & $10(9.17)$ \\
Warfarin & $9(8.26)$ \\
Furosemide & $8(7.34)$ \\
Nitroglycerine & $8(7.34)$ \\
Atorvastatin & $7(6.42)$ \\
Aspirin & $7(6.42)$ \\
Perindopril & $5(4.59)$ \\
Rosuvastatin & $5(4.59)$ \\
Valsartan & $5(4.59)$ \\
Losartan & $4(3.67)$ \\
Enoxaparin & $4(3.67)$ \\
\hline
\end{tabular}

Table 4: Suspected adverse drug reactions.

\begin{tabular}{cc}
\hline Suspected ADR & No. (\%) of ADRs \\
\hline Bradycardia & $11(10.09)$ \\
Hypotension & $9(8.26)$ \\
Elevated serum creatinine & $9(8.26)$ \\
Electrolyte imbalance & $8(7.34)$ \\
Constipation & $8(7.34)$ \\
Raised INR & $5(4.59)$ \\
Abdominal discomfort & $5(4.59)$ \\
Vomiting & $5(4.59)$ \\
Tachycardia & $4(3.67)$ \\
Dry Cough & $4(3.67)$ \\
Elevated liver enzymes & $4(3.67)$ \\
Headache & $3(2.75)$ \\
Gastrointestinal haemorrhage & $3(2.75)$ \\
Pedal oedema & $3(2.75)$ \\
\hline
\end{tabular}

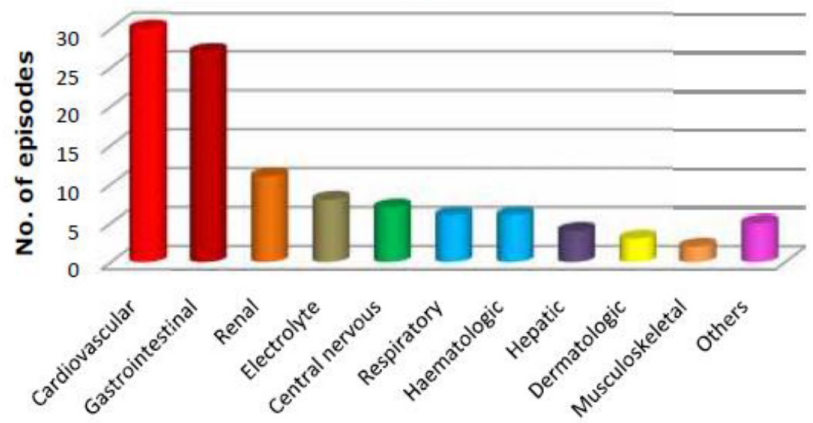

Figure 2: Organ system affected by ADRs.

Indian Journal of Pharmacy Practice, Vol 11, Issue 2, Apr-Jun, 2018 


\section{Table 5: Management and outcome of ADRs}

\begin{tabular}{cccc} 
Management & $\begin{array}{c}\text { No. (\%) of } \\
\text { ADRs }\end{array}$ & Outcome & $\begin{array}{c}\text { No. (\%) of } \\
\text { ADRs }\end{array}$ \\
\hline Drug withdrawn & $45(41.28)$ & Fatal & $0(00)$ \\
Drug withheld & $10(9.17)$ & Recovery & $60(83.33)$ \\
Dose altered & $11(10.09)$ & Continuing & $6(5.5)$ \\
No change & $37(33.95)$ & Unknown & $6(5.5)$ \\
Unknown & $6(5.51)$ & & \\
\hline
\end{tabular}

\section{Table 6: Causality assessment of ADRs}

Causality parameters

Certain

Probable

Possible

Unlikely

*WHO Probability scale.

\section{Table 7: Severity assessment of ADRs}

\begin{tabular}{cc} 
Severity parameters & No. (\%) of ADRs \\
\hline Mild & $58(53.21)$ \\
Moderate & $41(37.61)$ \\
Severe & $10(9.17)$ \\
\hline
\end{tabular}

* Hartwig et al. scale.

\begin{tabular}{cc}
\hline \multicolumn{2}{|c|}{ Table 8: Preventability assessment of ADRs* } \\
\hline Preventability parameters & No. (\%) of ADRs \\
\hline Definitely preventable & $9(8.25 \%)$ \\
Probably preventable & $8(7.25 \%)$ \\
Not preventable & $92(84.5 \%)$ \\
\hline
\end{tabular}

* Modified Schumock and Thornton scale.

\section{Severity assessment}

On severity assessment, it was found that $53 \%$ of ADRs were of mild severity, 41 (38\%) ADRs were moderate and only $9 \%$ of ADRs were severe in nature (Table 7 ).

\section{Preventability assessment}

On evaluation, it was observed that $84.5 \%$ of ADRs were not preventable. Nine (8.25\%) ADRs were definitely preventable and $8(7.25 \%)$ were probably preventable (Table 8).

\section{Predisposing factors}

Polypharmacy and age was the most common predisposing factor for ADRs. Polypharmacy was observed to be statistically significant risk factor for patients who developed ADRs $(\mathrm{r}=0.817, \mathrm{p}<0.01)$.

\section{DISCUSSION}

Adverse drug reactions (ADRs) are considered as one of the major public health concern which may contribute for increase healthcare burden. With a view to estimate the incidence of ADRs, the present study was conducted in the cardiology ward of Ibrahim Bin Hamad Obaidallah hospital, Ras Al Khaimah, UAE.

A total of 309 patients who were on cardiovascular drugs were enrolled in the present study. The overall incidence was found to be $23.3 \%$ which is in compliance with the pilot study conducted earlier. ${ }^{22}$ This observation is consistent with the incidence $(24.2 \%)$ reported in previous studies. ${ }^{23}$ There are also reports stating a higher incidence of ADRs in contrast with the present findings. ${ }^{24-25}$

In $3.88 \%$ of cases, patients were hospitalized due to ADRs which was supported by a study conducted by Arulmani et al. (3.4\%). ${ }^{26}$ However, a higher incidence $(6.4 \%)$ was seen in a study carried out by Kaur et al. ${ }^{18}$

There were 60 patients $(19.42 \%)$ who developed a total of 93 ADRs during their hospital stay. These findings were not comparable to that of a study conducted earlier $(10.9 \%) .{ }^{1}$ This could be due to different healthcare settings, variation in the pattern of drug usage and complexity of regimen.

Male preponderance over female $(24.9 \%$ vs. $21.2 \%)$ was observed in the present study, however the difference was not statistically significant $(\mathrm{p}=0.183)$. These findings are consistent with a study conducted by Rohit $e t$ al. where majority of ADRs were reported in men (62.1\%). ${ }^{27}$ On the contrary, higher incidence of ADRs in female was observed in studies conducted by Rodenburg et al. and Kaur et al. showing incidence of $57 \%$ and $27.9 \%$ respectively. ${ }^{18,28}$ The discrepancy in the present study could be due to different healthcare settings and variation in the disease pattern.

Considering the age group of patients, older patients ( $\geq 60$ years) were observed to have a higher number of ADRs $(n=73)$ in contrast with other age groups. However, there was no significant difference between the age group $(\mathrm{p}=0.601)$. This observation is comparable to that of a study conducted in United Kingdom by Kongkaew et al. which showed higher incidence of ADRs $(10.7 \%)$ in elderly than other age groups. ${ }^{24}$ Another study conducted by Kaur et al. also supports the above findings $(24.9 \%) .{ }^{18}$ However, there are also reports to show a higher incidence of ADRs between the age of 51- 60 years unlike that of the present observation. ${ }^{17}$ Similarly, Kurshid et al. observed that the most vulnerable age group for ADRs was $41-50$ years. $^{29}$

Considering patient's nationality, nationals experienced 57 ADRs while 52 ADRs were observed in expatriates. Among expatriates' patients, Indians accounted for the higher number of ADRs compared to that of others. This result is comparable to that of a study conducted by 
Abdulrazzaq et al. in Malaysia which showed that more Indians were likely to develop ADRs due to statins than other nationalities. ${ }^{30}$

In the present study, the mean length of hospital stays (LOS) for patients who developed ADRs was 10.11 days. This observation is comparable to that of a study conducted earlier (9.3 days). ${ }^{31}$ Similarly, a study conducted in a medical ICU in Korea by Park et al. revealed a positive correlation between patients who developed ADRs and their length of hospital stay. ${ }^{32}$ Dissimilar finding was also seen in a longitudinal observational study (4.2 days vs. 3.9)..$^{33}$

A positive correlation was established between the number of drugs (polypharmacy) and occurrence of ADRs which was found to be statistically significant $(p<0.01)$. The finding of the present study is comparable to that of earlier studies where polypharmacy was the only predictor for ADRs. ${ }^{25,34}$

On evaluation of drug class implicated in ADRs, betablockers $(14.68 \%)$ were the most common drug class followed by Renin-Aldosterone-Angiotensen-Receptors (RAAS) blockers and anti-coagulants. The present findings are similar to the findings reported in studies conducted by Haile et al. and Chan et al. ${ }^{3,31}$

In contrast to these findings, a study conducted by Rohit et al. reported that calcium channel blockers were the most common drug class involved in ADRs. ${ }^{27}$ Similarly, Runciman $e t a l$. showed that anticoagulant was the most implicated drug class with ADRs. ${ }^{35}$ The discrepancy in the present study could be due to recruitment of all kind of patients including cardiac conditions and other medical conditions.

In the present study, bradycardia was the most commonly observed ADR. Frequent use of bisoprolol in patient population which is known to cause bradycardia, might have influenced the incidence of bradycardia in the present study. The reaction observed in the present study is not consistent with that of studies conducted by Davies et al. and Chan et al. which showed electrolyte disturbances and postural hypotension as the most prevalent ADR. ${ }^{2,31}$

With regard to the organ system, cardiovascular system was the most affected one $(27.53 \%)$ followed by gastrointestinal $(24.77 \%)$. This observation is comparable to a study carried out by Wadhwa et al. where cardiovascular system was the most affected organ system by ADRs (47.69\%). ${ }^{5}$ However, the present findings are not comparable with that of a study carried out by
Khurshid et al. which showed central nervous system as the most commonly affected organ system..$^{29}$ This discrepancy in the findings could be due to variation in the pattern of drug use.

The frequent use of cardiovascular drugs in the present study mainly affecting the cardiovascular system might have shown the higher incidence of ADRs related to cardiovascular system. Regarding the management approach for ADRs, suspected drug was withdrawn in $41.28 \%$ of reactions. This finding is in support with studies conducted by Mateti et al. and Kaur et al. which demonstrated the withdrawal of suspected drug in most of the cases $(78.33 \% ; 47.5 \%) .{ }^{18,36}$ A study conducted by Gholami et al. showed that $65.6 \%$ cases required no additional treatment or change in dose of the offending drug. ${ }^{37}$ In another study by Wadhwa et al. $75 \%$ of ADRs were managed by temporary discontinuation of the drug. ${ }^{5}$ Evaluation of the outcome of ADRs suggested that in $83.33 \%$ cases, there was complete recovery from the reaction, but in $5.5 \%$ of the reactions no improvement was observed. Only six cases were either discharged against medical advice (AMA) or shifted to another hospital and therefore outcome was unknown.

The findings of the present study regarding outcome $(83.33 \%)$ is consistent with that of a study conducted earlier $(93.33 \%){ }^{36}$

On causality assessment of ADRs, majority of reactions were possible $(73 \%)$ in nature followed by probable $(17 \%)$. These results are compliance with the finding suggested by Karimzadeh et al. (62.86\%) and Khurshid et al. (57\%) which showed majority of reactions to be possible in nature. ${ }^{29,38}$ However, a study conducted by Chan et al. showed more of probable ADRs (41.4\%). ${ }^{31}$ This discrepancy in the present study could be due to different healthcare settings and types of ADRs reported.

Severity assessment of ADRs showed high incidence of mild reactions (53\%) in the present study. This observation is similar to that of studies carried out by Haile et al. (47.7\%) and Khurshid et al. (66.6\%), where reactions of mild severity were more common., ${ }^{3,29}$ Dissimilar findings were also reported in a study conducted by Davies et al. where ADRs of moderate severity were most common $(76.9 \%){ }^{2}$ The discrepancy could be due to different healthcare settings and more intensive approach for high risk group patients.

Preventability assessment of ADRs showed 15.5\% of preventable ADRs in the present study which is comparable with that of findings suggested by Wadhwa 
et al. which showed $16.14 \%$ of preventable ADRs. ${ }^{5}$ However, the percentage of preventable ADRs $(76.7 \%)$ observed in a study conducted by Chan et al. was high compared to the present study. ${ }^{31}$

This was a prospective observational study and all patients admitted during the study period were enrolled. There was no randomization done for the study. The design and duration of the study restrict to generalize the findings as the study involved limited number of patients.

It was difficult to homogenize the population demographically and therefore, more number of male patients was enrolled during the study period.

There was no follow-up for recruited patients after discharge. Hence, information pertaining to occurrence of ADRs due to maintenance treatment is lacking.

The present study demonstrated high incidence of ADRs among patients on cardiovascular drugs. The design and duration of the study restrict to generalize the findings. However, intensive approach towards monitoring and reporting of ADRs could help healthcare professionals in minimizing preventable ADRs.

\section{CONCLUSION}

The present study demonstrated high incidence of adverse drug reactions in patients on cardiovascular drugs. Demographically, higher incidence was observed in males and patients above 60 years of age. Beta-blockers were the most common drug class implicated in causing ADR especially bisoprolol. The most common suspected ADR observed during the study was bradycardia, most probably due to the use of bisoprolol. Cardiovascular system was one of the most prominent organ system affected by drugs. Polypharmacy was found to be the most important predisposing factor for ADRs. In majority of cases, suspected drug was withdrawn which in turn led to recovery of patients. Most of the reactions were possible in causality, mild in severity and not preventable. High incidence of ADRs insists for vigilant monitoring to prevent further recurrence. Intervention by clinical pharmacists might improve the reporting and monitoring aspects of ADRs.

\section{ACKNOWLEDGEMENT}

The authors wish to thank RAK Medical and Health Sciences University, Ras Al Khaimah, UAE and Ibrahim Bin Hamad Obaidallah Hospital, Ras Al Khaimah, UAE for their constant support and cooperation in the successful completion of this study. We also thank Dr. Sathvik B. Sridhar for his support extended towards statistical evaluation of study data.

\section{CONFLICT OF INTEREST}

The authors declare no conflict of interest.

\section{ABBREVIATIONS}

ADRs: Adverse Drug Reactions, AHA: American Heart Association, CVD: Cardiovascular Disease, DDI: Drug-Drug Interaction, INR: International Normalized Ratio, RAAS: Renin Angiotensin Aldosterone System, UAE: United Arab Emirates, WHO: World Health Organization.

\section{SUMMARY}

High incidence of ADRs due to cardiovascular drugs insists for vigilant monitoring by healthcare professionals to prevent the further recurrence. Intervention by clinical pharmacists can improve the monitoring and reporting aspects of ADRs.

\section{REFERENCES}

1. Lazarou J, Pomeranz $\mathrm{BH}$, and Corey $\mathrm{PN}$ et al. Incidence of adverse drug reactions in hospitalized patients: A meta-analysis of prospective studies. JAMA. 1998;279(15):1200-5.

2. Davies EC, Green CF, Taylor S, Williamson PR, Mottram DR, and Pirmohamed $\mathrm{M}$ et al. Adverse drug reactions in hospital in-patients: A prospective analysis of 3695 patient-episodes. PLoS One. 2009;4(2):e4439.

3. Haile DB, Ayen WY, and Tiwari P et al. Prevalence and assessment of factors contributing to adverse drug reactions in wards of a tertiary care hospital, India. Ethiop J Health Sci. 2013;23(1):39-48.

4. Schneeweiss S, Hasford J, Gottler M, Hoffmann A, Riethling AK, and Avorn $\mathrm{J}$ et al. Admissions caused by adverse drug events to internal medicine and emergency departments in hospitals: A longitudinal population-based study. Eur J ClinPharmacol. 2002;58:285-91.

5. Wadhwa T, Patil PA, and Suresh VP et al. Monitoring Adverse Drug Reactions in Coronary Thrombosis Patients Admitted to Intensive Cardiac Care Unit in a Tertiary Care Hospital. Indian Journal of Pharmacy Practice. 2013;6(1):6-12.

6. Bates DW, Cullen DJ, Laird N, Petersen LA, Small SD, and Servi D et al. Incidence of Adverse Drug Events and Potential Adverse Drug Events: Implications for Prevention. Journal of American Medical Association JAMA. 1995;274(1):29-34.

7. Classen DC, Pestotnik SL, and Evans RS et al. Adverse drug events in hospitalized patients. JAMA. 1997;277(4):301-6.

8. Thomas EJ, Studdert DM, and Newhouse JP, et al. Costs of medical injuries in Utah and Colorado. Inquiry. 1999;36(3):255-64.

9. Bates DW, Spell N, and Cullen DJ, et al. The costs of adverse drug events in hospitalized patients. JAMA 1997;277(4):307-11.

10. Stephens M. Introduction to pharmacovigilance. In: Talbot J and Waller P (eds.) Stephens' detection of new adverse drug reactions. $5^{\text {th }}$ ed. John Wiley and Sons, Ltd, West Sussex, England. 2007;1-90.

11. Sharma S, Sharma J, and Aggarwal T et al. A survey on knowledge and perception of pharmacy students towards adverse drug reaction (ADR) reporting. Asian J Pharm Clin Res. 2012;5(3):129-130.

12. Srinivasan $R$, and Ramya $G$ et al. Adverse drug reaction-causality assessment. IJRPC. 2011;1(3):606-610. Available online at www.ijrpc.com

13. Wilbu K. Pharmacovigilance in the Middle East: a survey of 13 arabic-speaking countries. Drug Saf. 2013;36(1):25-30 
14. National Cardiovascular Disease Surveillance. Available from: http://www.cdc. gov/DHDSP/ncvdss/index.htm (Accessed on September 10,2013)

15. Cardiovascular research. Available from: http://www.centenary.org.au/p/ ourresearch/cardiovascular/(Accessed on March 04,2014)

16. The shifting burden of cardiovascular disease in Australia. Available from: http://www.heartfoundation.org.au/sitecollectiondocuments/hf-shifting_burdencvd-accecons-2005-may.Pdf (Accessed on March 04,2014)

17. Aparasus RR. Visits to office-based physicians in the United States for medication-related morbidity. J Am Pharm Assoc. 1999;39(1):332-337.

18. Kaur S, Kapoor V, Mahajan R, Lal M, and Gupta S et al. Monitoring of incidence, severity, and causality of adverse drug reactions in hospitalized patients with cardiovascular disease. Indian Journal of Pharmacology. 2011;43(1):22-26.

19. World health organization collaboration centre for International drug monitoring. The importance of pharmacovigilance. Safety monitoring of medicinal products. Geneva: world health organization. 2002.

20. Hartwig SC, Siegel J, Schneider PJ. Preventability and severity assessment in reporting adverse drug reactions. Am J Hosp Pharm. 1992;49:2229-32.

21. Schumock GT, Thornton JP. Focusing on the preventability of adverse drug reactions. Hosp Pharm. 1992;27:538.

22. El Sheikh SG, Rao PG, Wadhwa T. Monitoring and reporting of adverse drug reactions due to cardiovascular drug in patients admitted to a secondary care hospital in Ras Al Khaimah, UAE. Paper presented at: Dubai International Pharmaceuticals and Technologies Conference and Exhibition - DUPHAT; 2014 March 10-12; Dubai, United Arab of Emirates.

23. Mohebbi N, Shalviri G, Salarifar M, Salamzadeh J, and Gholami K et al. Adverse drug reactions induced by cardiovascular drugs in cardiovascular care unit patients. Pharmacoepidemiol Drug Saf. 2010 Sept;19(9):889-94.

24. Kongkaew C, Noyce PR, and Ashcroft DM. Hospital admissions asscosiated with adverse drug reactions: a systematic review of prospective observational studies. Ann Pharmacother. 2008;42(7):1017-25.

25. Mugosa S, Todororvic Z, and Zaimovic MS. Adverse drug reactions and polypharmacy in cardiac patients. BMC Pharmacology and Toxicology. 2012;13(1):A39.

26. Arulmani R, Rajendran SD and Suresh B. Adverse drug reaction monitoring in a secondary care hospital in South India. $\mathrm{Br} \mathrm{J}$ ClinPharmacol. Blackwell Publishing Ltd 2007;65(2):210-216.

27. Rohit S, Khaleel A, and Santani DD. Reporting and monitoring of adverse drug reactions with cardiac drugs. International Research Journal of Pharmacy (IRJP). 2011;2(7):116-9
28. Rodenburg EM, Stricker BH and Visser LE. Sex-related differences in hospital admissios attributed to adverse drug reactions in the Netherlands. $\mathrm{Br} J$ ClinPharmacol. 2010;71(1):95-104.

29. Khurshid F, Aqil M, Alam MS, Kapur P, and Pillai K. Monitoring of adverse drug reactions associated with antihypertensive medicines at a university teaching hospital in New Delhi. DARU Journal of Pharmaceutical Sciences. 2012;20(1):34.

30. AbdulRazzaq HA, Abd Aziz N, Hassan Y, Kassan YW, and Ismail O. Predictors of the Common Adverse Drug Reactions of Statins, Dyslipidemia - From Prevention to Treatment, Kelishadi R (Ed.), InTech 2012, Available from: http://www.intechopen.com/books/dyslipidemia-from-prevention-to-treatment/ predictors-of-the-commonadverse-drug-reactions-of-statins

31. Chan M, Nicklason F, and Vial JH. Adverse drug events as a cause of hospital admission in the elderly. Internal Medicine Journal. 2001;31(1):199-205.

32. Park S, In Y, Suh GY, Sohn K, and Kim E. Evaluation of adverse drug reactions in medical intensive care units. Eur J ClinPharmacol. 2013;69(1):119-31.

33. Tumwikirize WA, Ogwal-Okeng JW, Vernby A, Anokbonggo WW, Gustafsson LL, and Lundborg SC. Adverse drug reactions in patients admitted on internal medicine wards in a district and regional hospital in Uganda. Afr Health Sci. 2011;11(1):72-78.

34. Lobo MG, Pinheiro SM, Castro JG, Momente VG, and Pranchevicius MS. Adverse drug reaction monitoring: support for pharmacovigillance at a tertiary care hospital in northern Brazil. BMC Pharmacology and Toxicology. 2013;14(1):1-7.

35. Runciman WB, Roughead EE, Semple SJ, and Adams RJ. Adverse drug events and medication errors in Australia. International Journal for Quality in Health Care. 2003; 15(1):i49-i59.

36. Mateti UV, Nekkanti H, Vilakkathala R, Rajakannan T, Mallayasamy S, and Ramachandran P. Pattern of angiotensin-converting enzyme inhibitors induced adverse drug reactions in South India teaching hospital. North American Journal of Medical Sciences. 2012;4(4):185-9.

37. Gholami K, Ziaie S, and Shalviri G et al. Adverse drug reactions induced by cardiovascular drugs in outpatients. Pharmacy Practice. 2008;6(1):51-55.

38. Karimzadeh I, Namazi S, Shalviri G, and Gholami K et al. Cardiovascular drug reactions in hospitalized patients in cardiac care unit. Afr J Pharm Pharmacol. 2011;5(4):493-9. 